\title{
Variety of critical state nature manifestations
}

\author{
E.D.Soldatova \\ Dnipropetrovsk State University, Department of Physics, \\ Dnipropetrovsk, Ukraine
}

Received November 23, 1998, in final form October 15, 1999

The thermodynamical method of studying the critical state is developed based on the constructive definition of the critical state by means of linear uniform equation systems and the study of the critical state stability criteria. It permits us to reveal the diversity of critical state nature manifestations and the uncertainties in the existing description of critical state thermodynamics related to this diversity. The existence of four basic alternative types of critical behaviour of one-component systems is established and described, as well as their classification is presented. The behaviour of the whole complex of thermodynamical parameters is investigated for each type of critical state. The method under consideration describes analytical and nonanalytical behaviour of thermodynamical quantities. The results of the investigations are illustrated by the known models and by experimental data.

Key words: critical state

PACS: $64.60 . F r$

\section{Introduction}

Description of the behaviour of thermodynamical parameters near the critical points is one of the basic tasks of the critical state theory. Strict statistical calculations connected with the evaluation of the statistical sum of realistic systems are unavailable at present because of the impossibility of exactly accounting for the interaction and, moreover, for the fluctuations which are large near the critical point. At the same time, large fluctuations and their correlations considerably complicate experimental measurements in the immediate vicinity of the critical point. These theoretical and experimental difficulties constitute the problem of critical state. Its solution is a vital task.

The main efforts of the theory were directed to the analysis of exactly solvable two-dimensional models and to the development of the hypotheses of scaling law and universality as well as the renormalization group approach in different versions [1-8]. 
However, many of their theses are hypothetical and are based on considering the Hamiltonian of a special type. The existence of realistic systems and consistent models in statistical mechanics which contradict the scaling and universality hypotheses, for example, the exactly solvable two-dimensional Lieb and Baxter models, is noteworthy by that. Probably, the modern theory of critical phenomena, in spite of its great achievements, does not cover the whole variety of critical states observed in real systems and the consistent statistical models including exactly solvable ones.

The problem of a possible critical behaviour of thermodynamic parameters should be solved based on the fundamental principles, without any kind of hypotheses, i.e. using the thermodynamical method which is characterized by fidelity and maximum generality of its results. Even within the thermodynamics of the critical state there exist some questions to be answered. First of all, these questions are associated with the uncertainties in the critical behaviour of some quantities, for example, heat capacity $C_{V}$ and sound velocity. Various attempts to ascertain their finite or divergent behaviour at the critical point have been based either on some admissions or erroneous proof, as mentioned in $[9,10]$. There is no synonymous conclusion concerning the behaviour of higher derivatives of potentials at this point and some other problems as well. The same holds for unsolvable uncertainties in differential equations of thermodynamics at the critical point, in particular, in the Plank-Gibbs equation [10]. No analytical proof is known for this equation by the time. It is characteristic that such uncertainties always arise when the problem is solved in a general way in terms of the conventional definition of the critical point:

$$
\left(-\frac{\partial P}{\partial V}\right)_{T}=0, \quad\left(-\frac{\partial^{2} P}{\partial^{2} V}\right)_{T}=0, \quad\left(-\frac{\partial^{3} P}{\partial^{3} V}\right)_{T}>0 .
$$

It is obvious that we encounter the fundamental problems of the critical state thermodynamics. The following questions should be answered: what is the origin of these uncertainties? Which parameters define the critical state? Aren't these uncertainties a consequence of the diversity of critical state nature manifestations revealed by experimental and model investigations? To solve the problem one has to broaden and deepen the existing thermodynamical approach, first of all to introduce a constructive definition of the critical state. It is natural to take the thermodynamic stability requirements as a principle of the investigations, since the system is under extremal conditions, i.e. at the border of its stability.

\section{On the stability of the thermodynamical equilibrium}

Let us consider a homogenous one-component system, the internal energy of which is a function of the independent variables $S$ (entropy) and $x$ (a generalized thermodynamical coordinate): $U=U(S, x)$. These coordinates correspond to the thermodynamical forces $T$ and $X$. Volume $V, \mathcal{M}$, electric polarization $\mathcal{P}$ can serve as $x$. The correspondent thermoforces would then be the pressure $(-P)$, the strength of magnetic $H$ and electric $E$ fields. 
Further consideration will be based on the fundamental Gibbs's work [11] without admitting hypotheses of any kind. According to [11], the general condition for stability of the system has the form:

$$
\Delta U-T \delta S-X \delta x>0
$$

where $\delta S, \delta x$ are an arbitrary (virtual) deviations of $S, x$. Expanding $\Delta U$ in a series on $\delta S, \delta x$ we write the condition (1) in the following way:

$$
\sum_{n=2}^{\infty} \frac{1}{n !}\left(\delta S \frac{\delta}{\delta S}+\delta x \frac{\delta}{\delta x}\right)^{n} U>0
$$

From here for small $\delta S, \delta x$ we infer the requirement for the stability of the homogenous system $[11,12]$ :

$$
\begin{gathered}
D=\frac{\partial(T, X)}{\partial(S, x)}=\left(\frac{\partial T}{\partial S}\right)_{x}\left(\frac{\partial X}{\partial x}\right)_{S}-\left(\frac{\partial T}{\partial x}\right)_{S}^{2} \geqslant 0 \\
\left(\frac{\partial T}{\partial S}\right)_{x} \geqslant 0 ; \quad\left(\frac{\partial X}{\partial x}\right)_{S} \geqslant 0 .
\end{gathered}
$$

Using the notations [14], let us call $D$ the stability determinant of the system; parameters $\left(\frac{\partial T}{\partial S}\right)_{x},\left(\frac{\partial X}{\partial x}\right)_{S},\left(\frac{\partial T}{\partial x}\right)_{S}$ measured under constant thermocoordinates are the adiabatic quantities (AQ's), the parameters $\left(\frac{\partial T}{\partial S}\right)_{X},\left(\frac{\partial X}{\partial x}\right)_{T},\left(\frac{\partial T}{\partial x}\right)_{X}$ measured under constant thermoforces are isodynamic quantities (IQ's). The parameters $\left(\frac{\partial T}{\partial S}\right)_{x}$, $\left(\frac{\partial X}{\partial x}\right)_{S}$ are the adiabatic stability coefficients (ASC's); parameters $\left(\frac{\partial T}{\partial S}\right)_{X},\left(\frac{\partial X}{\partial x}\right)_{T}$ are isodynamic stability coefficients (ISC's). $D$, ASC's and ISC's are the principal characteristics of stability of a system. The thermodynamic transformations enable us to establish the relations between them:

$$
\begin{gathered}
D=\frac{\partial(T, X)}{\partial(S, x)}=\left(\frac{\partial T}{\partial S}\right)_{x}\left(\frac{\partial X}{\partial x}\right)_{S}-\left(\frac{\partial T}{\partial x}\right)_{S}^{2} \\
=\left[\left(\frac{\partial T}{\partial S}\right)_{X}^{-1}\left(\frac{\partial X}{\partial x}\right)_{T}^{-1}-\left(\frac{\partial T}{\partial x}\right)_{X}^{-2}\right]^{-1}, \\
D=\left(\frac{\partial T}{\partial S}\right)_{X}\left(\frac{\partial X}{\partial x}\right)_{S}=\left(\frac{\partial T}{\partial S}\right)_{x}\left(\frac{\partial X}{\partial x}\right)_{T}=\left(\frac{\partial T}{\partial x}\right)_{X}\left(-\frac{\partial T}{\partial x}\right)_{S} .
\end{gathered}
$$

At the critical point we have:

$$
D=0, \quad\left(\frac{\partial T}{\partial S}\right)_{x} \geqslant 0, \quad\left(\frac{\partial X}{\partial x}\right)_{S} \geqslant 0, \quad\left(\frac{\partial T}{\partial S}\right)_{X} \geqslant 0, \quad\left(\frac{\partial X}{\partial x}\right)_{T} \geqslant 0 .
$$

The condition $D=0$ was first investigated by Gibbs.

The object of our investigation is the possible types of behaviour of the whole complex of thermodynamical characteristics (AQ's and IQ's). 
In the vicinity of critical points these quantities behave in different ways. If the behaviour of IQ's, for example, $\left(\frac{\partial P}{\partial V}\right)_{T},\left(\frac{\partial T}{\partial V}\right)_{P},\left(\frac{\partial T}{\partial S}\right)_{P}=\frac{T}{C_{p}}$ is known, there is uncertainty concerning the behaviour of AQ's, for example $\left(-\frac{\partial P}{\partial V}\right)_{S},\left(\frac{\partial T}{\partial V}\right)_{S},\left(\frac{\partial T}{\partial S}\right)_{V}=$ $\frac{T}{C_{V}}$.

Using the relations (3)-(5), we can also state that if $D=0$, then, at least, three quantities, one from each pair (4), will be zero, moreover one of them will be one (and possibly both) of the ISC's. This information exhausts the condition $D=0$.

Thus, neither by means of the traditional definition of the critical state nor from Gibbs's condition $D=0$ one can completely describe behaviour of AQ's and IQ's and solve the problems under consideration. That is why introducing a more profound definition of the critical state is so vital.

\section{Definition of the critical state}

At the critical point the limit properties of heterogenous and homogenous states are combined in a noncontradictory way. Indeed, the critical point is a limit point of the phase equilibrium line (the binodale), which is defined by the Clapeyron-Clausius equation. For the first type phase transitions we have

$$
\left[\begin{array}{c}
\Delta T \\
\Delta X
\end{array}\right]=\left[\begin{array}{l}
0 \\
0
\end{array}\right], \quad\left[\begin{array}{c}
\Delta S \\
\Delta x
\end{array}\right] \neq\left[\begin{array}{l}
0 \\
0
\end{array}\right], \quad\left(\frac{\mathrm{d} X}{\mathrm{~d} T}\right)_{L}=-\frac{\Delta S}{\Delta x},
$$

where $\Delta$ is the binodale increment along the phase connode (isotherm, isobar or any other isoforce). In the critical point limit $(\Delta \rightarrow d)$ these conditions take the form:

$$
\left[\begin{array}{l}
\mathrm{d} T \\
\mathrm{~d} X
\end{array}\right]=\left[\begin{array}{l}
0 \\
0
\end{array}\right], \quad\left[\begin{array}{l}
\mathrm{d} S \\
\mathrm{~d} x
\end{array}\right] \neq\left[\begin{array}{l}
0 \\
0
\end{array}\right], \quad\left(\frac{\mathrm{d} X}{\mathrm{~d} T}\right)_{\mathrm{cr}}=-\frac{\mathrm{d} S}{\mathrm{~d} x} .
$$

On the other hand, the critical point is an equilibrium homogenous state and it permits one to write the definition of the critical state as a linear homogenous equation system:

$$
\left.\begin{array}{c}
\mathrm{d} T=\left(\frac{\partial T}{\partial S}\right)_{x} \mathrm{~d} S+\left(\frac{\partial T}{\partial x}\right)_{S} \mathrm{~d} x=0 \\
\mathrm{~d} X=\left(\frac{\partial X}{\partial S}\right)_{x} \mathrm{~d} S+\left(\frac{\partial X}{\partial x}\right)_{S} \mathrm{~d} x=0
\end{array}\right\} \quad \text { at } \quad\left(\frac{\mathrm{d} X}{\mathrm{~d} T}\right)_{\text {cr }}=-\frac{\mathrm{d} S}{\mathrm{~d} x}
$$

A nonzero solution of the equation system exists if

$$
\operatorname{Det}\left[\begin{array}{cc}
\left(\frac{\partial T}{\partial S}\right)_{x} & \left(\frac{\partial T}{\partial x}\right)_{S} \\
\left(\frac{\partial T}{\partial x}\right)_{S} & \left(\frac{\partial X}{\partial x}\right)_{S}
\end{array}\right]=D=0
$$

The condition coincides with the well-known condition of the critical state and, in general, is valid along the whole spinodale.

The solution of the system $-\mathrm{d} S / \mathrm{d} x$ which is the limit slope of the phase equilibrium line at the critical point $\left(K_{\mathrm{c}}\right)$ permits us to select just the critical point on 
the spinodale. On the other hand, we can express the solution via the AQ's and the ASC's at the critical point itself when using the condition $D=0$.

$$
-\frac{\mathrm{d} S}{\mathrm{~d} x}=K_{\mathrm{c}}=\left[\operatorname{sign}\left(\frac{\partial T}{\partial x}\right)_{S}\right] \sqrt{\frac{(\partial X / \partial x)_{S}}{(\partial T / \partial S)_{x}}} .
$$

Thus, the introduced definition $(6,7)$ describes the critical state in terms of the AQ's via a system of linear homogenous equations and demonstrates that the basic quantities, defining the critical state, are the ASC's and the slope of the phase equilibrium line at the critical point $K_{\mathrm{c}}$ connected with the ASC's. The definition successfully describes the critical state of a liquid-vapour system, the $\gamma \leftrightarrow \alpha$ transition in metallic cerium, ferromagnetics, ferroelectrics and other systems.

To study the critical behaviour of a system, the definition $(6,7)$ should be combined with the conditions of the critical state stability.

\section{The conditions of the critical state stability}

Let us consider the stability condition (2) written in terms of moments of the expansion of $U$ over arbitrary variations of $\delta S, \delta x$. A necessary condition of stability is imposed on the least moment of the expansion $\delta^{2} U$.

At the critical point, as a consequence of feasibility of the equality $D=0$, it can be expressed explicitly in terms of the ASC's:

$$
\delta^{2} U=\left[\sqrt{\left(\frac{\partial T}{\partial S}\right)_{x}} \delta S+\left[\operatorname{sign}\left(\frac{\partial T}{\partial x}\right)_{S}\right] \sqrt{\left(\frac{\partial X}{\partial x}\right)_{S}} \delta x\right]^{2} \geqslant 0 .
$$

Further consideration deals with the investigation of the critical state stability itself. From (8) it follows that there exist such $\delta S^{\prime}, \delta x^{\prime}$ among arbitrary $\delta S, \delta x$ that satisfy the condition

$$
\left(\frac{\partial T}{\partial S}\right)_{x}^{1 / 2} \delta S^{\prime}=-\left[\operatorname{sign}\left(\frac{\partial T}{\partial x}\right)_{S}\right]\left(\frac{\partial X}{\partial x}\right)_{S}^{1 / 2} \delta x^{\prime}
$$

and for which $\delta^{2} U\left(\delta S^{\prime}, \delta x^{\prime}\right)=0$. The next moment of the expansion is $\delta^{3} U\left(\delta S^{\prime}, \delta x^{\prime}\right)=$ 0 since the sign of $(2)$ is constant and one has to consider the condition $\delta^{4} U\left(\delta S^{\prime}, \delta x^{\prime}\right) \geqslant$ 0 with $\sum_{n=4}^{\infty} \frac{1}{n !} \delta^{n} U\left(\delta S^{\prime}, \delta x^{\prime}\right)>0$. It is connected with the fact that $\delta S$ and $\delta x$ can change their signs simultaneously, thus causing the change of $\delta^{3} U$ sign. Consequently the stability conditions of the critical state are determined by the correlations:

$$
\begin{gathered}
\frac{\partial}{\partial S}\left(\frac{\partial T}{\partial S}\right)_{x} K_{\mathrm{c}}^{3}-3 \frac{\partial}{\partial x}\left(\frac{\partial T}{\partial S}\right)_{x} K_{\mathrm{c}}^{2}+3 \frac{\partial}{\partial x}\left(\frac{\partial X}{\partial x}\right)_{S} K_{\mathrm{c}}-\frac{\partial}{\partial x}\left(\frac{\partial X}{\partial x}\right)_{S}=0 \\
\frac{\partial^{2}}{\partial S^{2}}\left(\frac{\partial T}{\partial S}\right)_{x} K_{\mathrm{c}}^{4}+4 \frac{\partial^{2}}{\partial x \partial S}\left(\frac{\partial T}{\partial S}\right)_{x} K_{\mathrm{c}}^{3}-6 \frac{\partial^{2}}{\partial x^{2}}\left(\frac{\partial T}{\partial S}\right)_{x} K_{\mathrm{c}}^{2}
\end{gathered}
$$




$$
+4 \frac{\partial^{2}}{\partial x \partial S}\left(\frac{\partial X}{\partial x}\right)_{S} K_{\mathrm{c}}-\frac{\partial^{2}}{\partial x^{2}}\left(\frac{\partial X}{\partial x}\right)_{S} \geqslant 0 ; \quad K_{\mathrm{c}}=-\frac{\delta S^{\prime}}{\delta x^{\prime}} .
$$

The signs ' in $\delta S^{\prime}$ and $\delta x^{\prime}$ will be omitted for convenience hereinafter.

The analysis shows that the critical state will be stable under the following conditions imposed on the ASC's and their derivatives:

1. $\left(\frac{\partial T}{\partial S}\right)_{x} \neq 0,\left(\frac{\partial X}{\partial x}\right)_{S} \neq 0, \quad\left(\delta S^{\prime} \neq 0, \delta x^{\prime} \neq 0\right)$

2. $\left(\frac{\partial T}{\partial S}\right)_{x} \neq 0,\left(\frac{\partial X}{\partial x}\right)_{S}=0, \quad\left(\delta S^{\prime}=0, \delta x^{\prime} \neq 0\right)$;

$\frac{\partial}{\partial x}\left(\frac{\partial X}{\partial x}\right)_{S}=0, \quad \frac{\partial^{2}}{\partial x^{2}}\left(\frac{\partial X}{\partial x}\right)_{S} \geqslant 0 ;$

3. $\left(\frac{\partial T}{\partial S}\right)_{x}=0,\left(\frac{\partial X}{\partial x}\right)_{S} \neq 0, \quad\left(\delta S^{\prime} \neq 0, \delta x^{\prime}=0\right)$;

$\frac{\partial}{\partial S}\left(\frac{\partial T}{\partial S}\right)_{x}=0, \quad \frac{\partial^{2}}{\partial S^{2}}\left(\frac{\partial T}{\partial S}\right)_{x} \geqslant 0$

4. $\left(\frac{\partial T}{\partial S}\right)_{x}=0,\left(\frac{\partial X}{\partial x}\right)_{S}=0, \quad\left(\delta S^{\prime}=0, \delta x^{\prime}=0\right)$;

$\frac{\partial}{\partial S}\left(\frac{\partial T}{\partial S}\right)_{x}=0, \quad \frac{\partial}{\partial x}\left(\frac{\partial T}{\partial S}\right)_{x}=0$

$\frac{\partial}{\partial S}\left(\frac{\partial X}{\partial x}\right)_{S}=0, \quad \frac{\partial}{\partial x}\left(\frac{\partial X}{\partial x}\right)_{S}=0$

$\frac{\partial^{2}}{\partial S^{2}}\left(\frac{\partial T}{\partial S}\right)_{x} \geqslant 0 ; \quad \frac{\partial^{2}}{\partial x^{2}}\left(\frac{\partial X}{\partial x}\right)_{S} \geqslant 0$.

It is important to emphasize that the existence of these four cases results from the equality $\delta^{2} U=0$ in which the coefficients are ASC's. By virtue of their molecularstatistical meaning (they are inversely proportional to the fluctuations of the corresponding parameters of the system $[11,12]$ ) these quantities may be either zero or nonzero, i.e. one does not encounter the divergence problem.

\section{Alternative types of the critical behaviour of the thermody- namic systems}

Let us consider the problem which is principal for the thermodynamics of the critical state - the behaviour of the whole complex of the thermodynamical characteristics (AQ's and IQ's). This problem is solved based on the introduced definition of the critical state $(6,7)$ and the critical state stability conditions that are imposed on the ASC's (9). Four types of critical behaviour are established. They correspond 
to the four cases of stability which are classified by the critical behaviour of the ASC's and the corresponding critical slope $K_{\mathrm{c}}$ of the phase equilibrium line.

$$
\begin{aligned}
& \text { 1. }\left(\frac{\partial T}{\partial S}\right)_{x} \neq 0, \quad\left(\frac{\partial X}{\partial x}\right)_{S} \neq 0 \Longrightarrow\left(\frac{\partial T}{\partial x}\right)_{S} \neq 0, \quad K_{\mathrm{c}} \neq\{0 ; \infty\} \text {; } \\
& K_{\mathrm{c}}=-\frac{d S}{d x}=\frac{(\partial T / \partial x)_{S}}{(\partial T / \partial S)_{x}}=\frac{(\partial X / \partial x)_{S}}{(\partial T / \partial x)_{S}} \\
& =\left[\operatorname{sign}\left(\frac{\partial T}{\partial x}\right)_{S}\right] \sqrt{\frac{(\partial X / \partial x)_{S}}{(\partial T / \partial S)_{x}}}=\left(\frac{\partial X}{\partial T}\right)_{x}=\left(\frac{\partial X}{\partial T}\right)_{S} \\
& =\lim _{D \rightarrow 0} \frac{(\partial X / \partial x)_{T}}{(-\partial T / \partial x)_{X}}=\lim _{D \rightarrow 0} \frac{(-\partial T / \partial x)_{X}}{(\partial T / \partial S)_{X}} \neq\{0 ; \infty\} .
\end{aligned}
$$

From $(3,4)$ it follows that at least three quantities should vanish at the critical point. Moreover, the ISC should be one of them. For this type of the critical behaviour, these quantities are all IQ's: $\left(\frac{\partial T}{\partial S}\right)_{X},\left(\frac{\partial X}{\partial x}\right)_{T},\left(\frac{\partial T}{\partial x}\right)_{X}$. Obviously all of them should tend to zero according to the same law, since $K_{\mathrm{c}} \neq\{0 ; \infty\}$.

$$
\begin{gathered}
\text { 2. }\left(\frac{\partial T}{\partial S}\right)_{x} \neq 0, \quad\left(\frac{\partial X}{\partial x}\right)_{S}=0 \Longrightarrow\left(\frac{\partial T}{\partial x}\right)_{S}=0, \quad K_{\mathrm{c}}=0 ; \\
K_{\mathrm{c}}=-\frac{\mathrm{d} S}{\mathrm{~d} x}=\frac{(\partial T / \partial x)_{S}}{(\partial T / \partial S)_{x}}=\left(\frac{\partial X}{\partial T}\right)_{x}=\lim _{D \rightarrow 0} \frac{(\partial X / \partial x)_{T}}{(-\partial T / \partial x)_{X}}=0 .
\end{gathered}
$$

The three quantities that necessarily vanish at the critical point are: $\left(\frac{\partial X}{\partial x}\right)_{S},\left(\frac{\partial X}{\partial x}\right)_{T}$, $\left(\frac{\partial T}{\partial x}\right)_{S}$.

$$
\begin{gathered}
\text { 3. }\left(\frac{\partial T}{\partial S}\right)_{x}=0, \quad\left(\frac{\partial X}{\partial x}\right)_{S} \neq 0 \Longrightarrow\left(\frac{\partial T}{\partial x}\right)_{S}=0, \quad K_{\mathrm{c}}=\infty ; \\
K_{\mathrm{c}}=-\frac{\mathrm{d} S}{\mathrm{~d} x}=\frac{(\partial X / \partial x)_{S}}{(\partial T / \partial x)_{S}}=\left(\frac{\partial X}{\partial T}\right)_{S}=\lim _{D \rightarrow 0} \frac{(-\partial T / \partial x)_{S}}{(\partial T / \partial S)_{X}}=\infty .
\end{gathered}
$$

The three quantities that necessarily vanish at the critical point are: $\left(\frac{\partial T}{\partial S}\right)_{x},\left(\frac{\partial T}{\partial S}\right)_{X}$, $\left(\frac{\partial T}{\partial x}\right)_{S}$.

$$
\begin{gathered}
\text { 4. }\left(\frac{\partial T}{\partial S}\right)_{x}=0, \quad\left(\frac{\partial X}{\partial x}\right)_{S}=0, \Longrightarrow\left(\frac{\partial T}{\partial x}\right)_{S}=0, \\
K_{\mathrm{c}}=? .
\end{gathered}
$$

In this case not only all AQ's but also the IQ's vanish, since they do not exceed the ASC's, and $K_{\mathrm{c}}$ is undefined.

Thus, we come to the conclusion that the behaviour of the thermodynamic quantities at the critical point is not uniform. There exist four fundamental types of the critical behaviour classified by the critical behaviour of the ASC's and the corresponding $K_{\mathrm{c}}$ (or by $K_{\mathrm{c}}$ and one of the ASC's). A specific definition of the critical 
behaviour enables us to determine the behaviour of the whole complex of the thermodynamical quantities (the AQ's and the IQ's), where the IQ's behaviour is a consequence of the AQ's behaviour in our approach.

To illustrate the realization of these types of the critical behaviour, we have analyzed numerous experimental data and a number of the known models. The result of the analysis are summarized in table 1 . The type of critical behaviour is ascertained according to the quantity $\left(\frac{\partial T}{\partial S}\right)_{x}=\frac{T}{C_{x}}$ (it is determined by the critical index $\alpha$ value and by the critical slope $K_{\mathrm{c}}$ ).

Let us pay attention to the Lieb and Baxter models considered in the monograph [2]. For ferroelectrics the "6-vertex" model of ice type (Lieb) gives the values of heat capacity index $\alpha^{\prime}=0\left(T \leqslant T_{\mathrm{c}}\right)$ and $\alpha=1\left(T \geqslant T_{\mathrm{c}}\right)$. In the both cases $\left(\frac{\partial T}{\partial S}\right)_{P}=0$ and at $K_{\mathrm{c}}=0$ we have the fourth type of the critical behaviour. The "8-vertex" Baxter model gives different values of heat capacity indexes including $\alpha=\alpha^{\prime}=2-(\pi / \mu)$. The parameter $\mu$ is connected with the interaction constant of the Hamiltonian and varies in the interval $(0, \pi)$ resulting in $\alpha$ values in the interval $(-\infty, 1)$. Hence, the ASC $\left(\frac{\partial T}{\partial S}\right)_{P}$ can take both zero and non-zero values. Therefore, this model may correspond both to the second and fourth types of critical behaviour.

These models are of special interest to the theory of critical state. The first one contradicts to the scaling law $\left(\alpha^{\prime} \neq \alpha\right)$ and the second one contradicts to the universality hypothesis since the indexes vary continuosly as a function of interaction parameters in it. Cases, when in the Lieb and Baxter models the scaling and universality hypotheses are broken are in good correspondence with the classification under consideration. There is a great number of experimental data on $\left(\frac{\partial T}{\partial S}\right)_{x}$ behaviour for liquids, ferromagnetics, and ferroelectrics. Their analysis and corresponding types of critical behaviour are shown in table. The critical $\gamma \leftrightarrow \alpha$ transition in metallic cerium is an exception. In our paper [14], the type of the critical behaviour of metallic cerium was ascertained based on the experimental fact [13] that the phase equilibrium line is a straight line with $K_{\mathrm{c}} \neq\{0, \infty\}$. We have shown [15] that all the thermodynamical quantities of cerium behave according to the first type of critical behaviour in our classification.

Let us discuss the uncertainties in thermodynamics of the critical state. It is obvious that the origin of the observed uncertainties is, first of all, the diversity of manifestations of the critical state nature. Establishing the four critical behaviour types removes the uncertainty in the AQ behaviour. Each type causes a different behaviour of the AQ's and consequently the IQ's. In particular, $C_{V}$ is finite in the first two types and is divergent in the third and in the fourth types. Let us discuss the feasibility of the Plank-Gibbs rule. It frequently includes a number of similar relations. It is common to emphasize the following one: $K_{\mathrm{c}}=\left(\frac{\partial P}{\partial T}\right)_{V}=\left(\frac{\partial P}{\partial T}\right)_{S}$. For our generalized system it takes the form:

$$
K_{\mathrm{c}}=\left(\frac{\partial X}{\partial T}\right)_{x}=\left(\frac{\partial X}{\partial T}\right)_{S} .
$$

It is obvious that it is satisfied unconditionally for the first type of critical behaviour, for the second and for the third types only parts from the relation can be 
Table 1. Types of critical behaviour for different models and experiments.

\begin{tabular}{|c|c|c|c|c|}
\hline $\mathrm{N}$ & Model, Substance & $K_{\mathrm{c}}$ & $\alpha, \alpha^{\prime}$ & $\begin{array}{c}\text { Type of } \\
\text { critical } \\
\text { behaviour }\end{array}$ \\
\hline & $\underline{\text { Models }}$ & & & \\
\hline 1. & Van-der-Waals & $\neq\{0, ; \infty\}$ & $\alpha=\alpha^{\prime}=0$ (finite) & 1 \\
\hline 2. & Curie-Weisse & 0 &,-- & 2 \\
\hline 3. & Ising $d=2$ & & $\alpha=\alpha^{\prime}=0$ & \\
\hline & ferromagnets & 0 & (log. div.) & 4 \\
\hline & lattice gas & not determ. & & 3,4 \\
\hline 4. & 6- vertex Lieb & 0 & $\alpha=0, \alpha^{\prime}=1$ & 4 \\
\hline 5. & 8-vertex Baxter & 0 & $\alpha=\alpha^{\prime}=\{-\infty ; 1\}$ & 2,4 \\
\hline 6. & Ising $d=3$ & & $\alpha=\alpha^{\prime}=0.125$ & \\
\hline & ferromagnets & 0 & & 3,4 \\
\hline & lattice gas & not determ. & & 3,4 \\
\hline 7. & Heisenberg $\mathrm{d}=3$ & 0 & $\begin{array}{c}\alpha=\alpha^{\prime}= \\
=\{-0.09 ;-0.20\}\end{array}$ & 2 \\
\hline & Experiment & & & \\
\hline 1. & Liquids & $\neq\{0, ; \infty\}$ & & 4 \\
\hline 2. & Ferromagnets & 0 & $\begin{array}{c}\alpha \simeq \alpha^{\prime}>0 \\
\alpha \simeq \alpha^{\prime}=0, \\
\alpha \simeq \alpha^{\prime}<0 \\
\text { may be } \alpha \neq \alpha^{\prime}\end{array}$ & 2,4 \\
\hline 3. & Ferroetoelectrics & 0 &,-- & 2,4 \\
\hline 4. & $\begin{array}{l}\text { Critical point of iso- } \\
\text { morphic } \gamma \leftrightarrow \alpha \text { transi- } \\
\text { tion in metallic } \\
\text { cerium }\end{array}$ & $\neq\{0, ; \infty\}$ & $\alpha=\alpha^{\prime}=0$ (finite) & 1 \\
\hline
\end{tabular}

satisfied. The behaviour of the first type $\left(K_{\mathrm{c}} \neq\{0 ; \infty\}\right.$ and finite ASC's) is observed, for example, in some liquids and in the critical $\gamma \leftrightarrow \alpha$ transition in metallic cerium. If the ASC's are zero, the fourth type takes place when $K_{\mathrm{c}}$ is arbitrary.

To prove the Plank-Gibbs rule is difficult, first of all, because of the existence of the fourth type which is alternative to the others. On the other hand, the uncertainty of $K_{\mathrm{c}}$ value in this type shows its ambiguity.

We connected the revealing of this uncertainty and a possible realization of the fourth type with the next approximation of the condition of the critical state stability. We shall call this approximation the extended method of the thermodynamical stability theory. 


\section{The extended method of the thermodynamical stability theory}

The necessity of this method arises, first of all, in the analysis of the conditions of the critical state stability in the case of zero values of all ASC's, resulting in the uncertainty of $K_{\mathrm{c}}$. According to (9), zero values of all ASC's mean zero values of their first partial derivatives, hence the conditions of stability should be imposed on the second partial derivatives of the ASC's in [17]. We have considered in details the case when some second partial derivatives of the ASC's were other than zero and the critical state stability was determined by the condition

$$
\delta^{4} U=\left(\delta S \frac{\delta}{\delta S}+\delta x \frac{\delta}{\delta x}\right)^{4} U \geqslant 0 .
$$

In this case the second partial derivatives of the ASC plays the part of characteristic quantities:

$$
\begin{gathered}
t_{s s}=\frac{\partial^{2}}{\partial S^{2}}\left(\frac{\partial T}{\partial S}\right)_{x} ; t_{s x}=\frac{\partial^{2}}{\partial S \partial x}\left(\frac{\partial T}{\partial S}\right)_{x} ; t_{x x}=\frac{\partial^{2}}{\partial x^{2}}\left(\frac{\partial T}{\partial S}\right)_{x} \\
m_{s x}=\frac{\partial^{2}}{\partial S \partial x}\left(\frac{\partial X}{\partial x}\right)_{S} ; m_{x x}=\frac{\partial^{2}}{\partial x^{2}}\left(\frac{\partial X}{\partial x}\right)_{S}
\end{gathered}
$$

The investigation of the critical stability type $\left(\delta^{4} U \geqslant 0, \delta^{4} U>0\right)$ yields:

1. If there are zeroes among $t_{s s}, m_{x x}$ then satisfied are the conditions:

$$
\begin{gathered}
t_{s s} \geqslant 0, \quad m_{x x} \geqslant 0, \quad t_{x x} \geqslant 0 \\
D_{1}=3 t_{x x} m_{x x}-2 m_{s s} \geqslant 0, \quad D_{2}=3 t_{s s} t_{x x}-2 t_{s x}^{2} \geqslant 0 .
\end{gathered}
$$

Moreover if $D_{1}=0$ then there exist $\delta S, \delta x$, such that

$$
\sqrt{6 t_{x x}} \delta S=-\left[\operatorname{sign} m_{s x}\right] \sqrt{m_{x x}} \delta x
$$

for which $\delta^{4} U=0$. Otherwise if $D_{2}=0$ then the equality $\delta^{4} U=0$ is true for $\delta S, \delta x$ such that

$$
\sqrt{6 t_{x x}} \delta x=-\left[\operatorname{sign} t_{s x}\right] \sqrt{t_{s s}} \delta S .
$$

For all other $\delta S$ and $\delta x$ the strict inequality $\delta^{4} U>0$ is satisfied.

2. If $t_{s s} \neq 0$ and $m_{x x} \neq 0$ then the characteristic quantities are the following functions:

$$
\begin{aligned}
p & =6\left[\frac{t_{x x}}{t_{s s}}-\left(\frac{t_{s x}}{t_{s s}}\right)^{2}\right], \quad q=4\left[2\left(\frac{t_{s x}}{t_{s s}}\right)^{3}-3 \frac{t_{x x} t_{s x}}{t_{s s}^{2}}+\frac{m_{s x}}{t_{s s}}\right], \\
r & =-3\left(\frac{t_{s x}}{t_{s s}}\right)^{4}+6 \frac{t_{x x} t_{s x}}{t_{s s}^{3}}-4 \frac{m_{s x} t_{s x}}{t_{s s}^{2}}+\frac{m_{x x}}{t_{s s}} \\
D_{0} & =\frac{64}{27} r\left[\left(\frac{p}{2}\right)^{2}-r\right]^{2}-q^{2}\left[\frac{1}{27} p\left(p^{2}-36 r\right)+\left(\frac{q}{2}\right)^{2}\right],
\end{aligned}
$$


where $D_{0}$ is the determinant of a fourth order equation. All these functions obey certain requirements imposed by the stability conditions $\delta^{4} U \geqslant 0$ and $\delta^{4} U>0$ [17].

Requirements imposed on the partial derivatives of ASC's and their functions by conditions of the critical state stability have been ascertained and the next approximation of the critical state conditions has been considered in the form of the set of uniform equations of the third order $[17,16]$

$$
\left\{\begin{aligned}
\mathrm{d}^{3} T & =\left(\mathrm{d} S \frac{\partial}{\partial S}+\mathrm{d} x \frac{\partial}{\partial x}\right)^{3} T=0 \\
\mathrm{~d}^{3} X & =\left(\mathrm{d} S \frac{\partial}{\partial S}+\mathrm{d} x \frac{\partial}{\partial x}\right)^{3} X=0
\end{aligned}\right.
$$

The set of equations (with taking into account the conditions of the state stability) has got from zero to two different values of $K_{\mathrm{c}}$ as physical solutions in dependence on the critical behaviour of the second partial derivatives of ASC's and their functions. According to it, the fourth type of the critical behaviour can be realized through five versions:

1. If $t_{s s}=0, m_{x x}=0, t_{x x}>0$ we have $K_{\mathrm{c}}^{(1)}=0, K_{\mathrm{c}}^{(2)}=\infty$.

2. If $t_{s s}=0, m_{x x}>0$ then $K_{\mathrm{c}}^{(1)}=\infty$ always. If $D_{1}=0$ and $t_{x x}>0$ then there also exists $K_{\mathrm{c}}^{(2)}=\frac{\operatorname{sign} m_{s x}}{\sqrt{6}}\left[\frac{m_{x x}}{t_{x x}}\right]^{1 / 2} \neq\{0 ; \infty\}$.

3. If $m_{x x}=0, t_{s s}>0$ then $K_{\mathrm{c}}^{(1)}=0$ always. If $D_{2}=0$ and $t_{x x}>0$ then there exists $K_{\mathrm{c}}^{(2)}=\frac{\sqrt{6}}{\operatorname{sign} t_{s x}}\left[\frac{t_{s s}}{t_{x x}}\right]^{1 / 2} \neq\{0 ; \infty\}$.

4. If $t_{s s}>0, m_{x x}>0$ there are two possibilities $K_{\mathrm{c}}^{(1,2)} \neq\{0 ; \infty\} \quad(q=0), K_{\mathrm{c}}^{(1)} \neq$ $\{0 ; \infty\} \quad(q \neq 0)$ depending on the values of $p, r, q$.

5. Possible is also the critical state which corresponds to the stability condition $\delta^{4} U>0$. It apparently is not an end of the phase transition line (the isolated critical point).

The case when the critical point has two distinct values of $K_{\mathrm{c}}$ is of special interest. For a simple one-component system under consideration (in accordance with the phase rule) this case should be observed at the point where two different lines of phase equilibrium meet. In the framework of our extended method, the description of a stable critical point where three different lines of phase equilibrium meet is possible (a critical triple point). In this case the stability condition $\delta^{6} U \geqslant 0$ and the set of equations $\left[\mathrm{d}^{5} T, \mathrm{~d}^{5} X\right]=[0,0]$ should be analyzed. Such a critical point exists, probably, in the exactly solvable Lieb's model of ferroelectric [18]. The violation of the scaling hypothesis in this model seems to be caused by the difference of critical behaviour types at $T<T_{\mathrm{c}}$ and $T>T_{\mathrm{c}}$.

The conditions of the critical state stability also give an opportunity to obtain some information about the asymptotic behaviour of thermodynamic quantities, first of all the AQ. It has been shown that the lowest and differing from zero derivative 
of a thermodynamical force over a thermodynamical coordinate is the derivative of the odd order $n$. For example, for the thermical ASC, nonvanishing derivatives are:

$$
\left(\frac{\partial T}{\partial S}\right)_{x},\left(\frac{\partial^{3} T}{\partial S^{3}}\right)_{x}, \ldots,\left(\frac{\partial^{n} T}{\partial S^{n}}\right)_{x}, \quad n=1,3,5, \ldots
$$

Let us consider the asymptotical behaviour of the quantities. We suppose $\left(\partial^{n} T / \partial S^{n}\right)_{x}=a \neq\{0 ; \infty\}$ and $\left(\partial^{k} T / \partial x^{k}\right)_{S}=0$ for $k<n$. It results in $\left|T-T_{\mathrm{c}}\right| \simeq$ $a\left|S-S_{\mathrm{c}}\right|^{n}$ at $x=x_{\mathrm{c}}$ in the vicinity of the critical point. Therefore

$$
\left(\frac{\partial S}{\partial T}\right)_{x}=C_{x} / T \simeq 1 / a_{n} \tau^{\frac{1}{n}-1}, \quad \tau=\left|T-T_{\mathrm{c}}\right| .
$$

Since the asymptotic behaviour of $C_{x}$ is determined by an index $\alpha$, we obtain $\alpha=1-1 / n$. Thus the sequence of nonvanishing derivatives with $n=1,3, \ldots \rightarrow \infty$ corresponds to the following sequence of the critical index values:

$$
\alpha=0, \frac{2}{3}, \ldots, \frac{n-1}{n}, \ldots \rightarrow 1 .
$$

The value $n=1$ corresponds to the finite $C_{x}$, for $n=3,5, \ldots C_{x}$ is divergent with fractional values of $\alpha$. Similar consideration can be applied to the other ASC's.

Let us note that the obtained values of $\alpha$ coincide with $\alpha$ of some known models. So $\alpha=0$ corresponds to the classical models, $\alpha=2 / 3-$ to 3 -dimensional spin model, $\alpha=1$ - to the Lieb's and Baxter's models.

Consequently the analysis of the asymptotical behaviour of the AQ shows that the extended method of the thermodynamical stability theory describes both analytic and nonanalytic behaviour.

\section{Conclusion}

With the developed thermodynamic method, the critical state of onecomponent systems has been studied on the basis of introducing the constructive definition of the critical state and investigating its stability. The following results were obtained:

1. It has been shown that the fundamental quantities determining the critical state were the adiabatic coefficients of stability (the ASC's) and the slope $K_{\mathrm{c}}$ of the phase equilibrium line at the critical point.

2. The existence of four fundamental alternative types of the critical state has been established. The types are classified by the critical behaviour of the ASC's and $K_{\mathrm{c}}$. For each type of the critical state the behaviour of the whole complex of the stability characteristics is analysed. The results of this analysis are illustrated by the known models and by experimental data.

3. Uncovering the diversity of the critical state, the method enables us to understand its origin and to reveal the existing uncertainties in the critical state thermodynamics. 
4. The possibility of the existence of a critical state of a new type has been shown, particularly, these are the critical points where 2 (or 3) phase equilibrium curves meet.

5. The analysis of the behaviour (including the asymptotical one) of the critical states fundamental characteristics shows that the method can describe both their analytical and nonanalytical behaviour.

\section{References}

1. Onsager L. Critical statistics. I. A two dimensional model with an order-disorder transition. // Phys. Rev., 1944, vol. 65, No. 1, p. 117-149.

2. Baxter R. Exactly Solvable Models in Statistical Mechanics. Academic Press, London, 1982.

3. Fisher M. The Nature of Critical Points. University of Colorado Press, Boulder, Colorado, 1965.

4. Widom B. Surface tension and molecular correlations near the critical point. Equation of state in the neighbourhood of the critical point. // J. Chem. Phys., 1965, vol. 43, No. 11, p. 3892-3905.

5. Kadanoff L.P., Gotze W., Hamblen D. Static phenomena near critical points: theory and experiment. // Rev. Mod. Phys., 1967, vol. 39, No. 2, p. 395-431.

6. Wilson K., Kogut J. The Renormalisation Group and $\varepsilon$-expansion. North-Holland Publishing Company, 1974.

7. Patashinskiy A.Z., Pokrovskiy V.L. Fluctuational Theory of Phase Transitions. Moscow, Nauka, 1982 (in Russian).

8. Yukhnovskiy I.R. Second Type Phase Transitions. Method of Collective Variables. Kiev, Naukova Dumka, 1985 (in Russian).

9. Bazarov I.P. Thermodynamics. Moscow, Vysshaya Shkola, 1983, 344 p.

10. Sychev B.B. Differential Equations of Thermodynamics. Moscow, Nauka, 1981 (in Russian).

11. Gibbs J.W. Thermodynamics. Statistical Mechanics. Moscow, Nauka, 1982 (in Russian).

12. Semenchenko V.K. Thermodynamical stability and phase transitions in crystals. // Crystallography, 1964, vol. 2, No. 5, p. 611-621 (in Russian).

13. Kutsar A.R $\gamma \leftrightarrow \alpha$ transition and volumetric abnormalities in cerium under pressure. // Doklady AN SSSR, 1979, vol. 245, No. 6, p. 1360-1364 (in Russian).

14. Soldatova E.D., Shvetz G.N. On thermodynamics of isomorphic $\gamma \leftrightarrow \alpha$ transition in metallic cerium. // Izvestiya vuzov. Fizika., 1991, vol. 5, p. 66-70 (in Russian).

15. Soldatova E.D., Shvetz G.N. Thermodynamical stability of cerium in the region of critical $\gamma \leftrightarrow \alpha$ transition. // Zhurnal Fiz. Him., 1990, vol. 64, No. 6, p. 1655-1657 (in Russian).

16. Soldatova E.D. Thermodynamical Stability in Critical State Region. Thesis for a degree of Doctor of Science (Physics and Matematics). Kyiv, KSU, 1991 (in Russian).

17. Soldatova E.D. Stability of critical state and asymptotical behaviour of thermodynamic quantities. // Zhurnal Fiz. Him., 1993, vol. 67, No. 9, p. 1434-1439. 
18. Soldatova E.D., Semenova O.A. Application of Lieb's model to description of critical state of one-component systems. // Jurnal Fiz. Him., 1993, vol. 67, No. 9, p. 1434-1439 (in Russian).

\title{
Різноманітність проявів природи критичного стану
}

\author{
Є.Д.Солдатова
}

Дніпропетровський державний університет, фізичний факультет, Дніпропетровськ

Отримано 23 листопада 1998 р., в остаточному вигляді 15 жовтня 1999 р.

Для вивчення критичного стану розвивається термодинамічний метод, який грунтується на конструктивному означенні критичного стану за допомогою системи лінійних однорідних рівнянь і вивченні критерію стабільності критичного стану. Це дозволяє виявити різноманітність проявів природи критичного стану і існуючі розходження у описі термодинаміки критичного стану, пов'язані з цією різноманітністю. Встановлено і описано існування чотирьох основних альтернативних типів критичної поведінки однокомпонентних систем, представлена їх класифікація. Для кожного типу критичного стану поведінка всього комплексу термодинамічних параметрів $€$ досліджена. Розглянутий метод описує аналітичну і неаналітичну поведінку термодинамічних величин. Результати дослідження ілюструються відомими моделями і експериментальними даними.

Ключові слова: критичний стан

PACS: $64.60 . F r$ 